\title{
FABIoT: A Flexible Agent-Based Simulation Model for IoT Environments
}

\author{
Marco Pérez-Hernández, Badraddin Alturki, Stephan Reiff-Marganiec \\ Department of Informatics, University of Leicester \\ Leicester, United Kingdom \\ $\{$ meph1, baba1, srm13\}@leicester.ac.uk
}

\begin{abstract}
The Internet of Things aims to digitize everyday physical objects by connecting them to the internet. As a result, cyber-physical environments of multiple sizes emerge, imposing new requirements on applications and software systems in regards support to heterogeneity and volatility. A challenging stage in the engineering of these systems is the validation. Although, there have been significant efforts to offer shared real-world testbeds, the simulations platforms are required to make the validation process cost and time effective. Existing simulation approaches only offer partial coverage to the key IoT environment characteristics, focus on communication or are specific for particular use cases and domains. In this paper, we propose a novel agent-based model that enables the simulation of the IoT systems with the key characteristics of an IoT environment. This model is designed to be flexible and adaptable to different experiments. Our approach introduces events in IoT environments as stochastic processes, enabling the evaluation of IoT systems under different conditions that otherwise would be time consuming and costly. We present the results of our experiments for evaluation of our model. These show that our proposal is a practical solution for the validation of IoT software systems, complementary to the real-world tests.
\end{abstract}

Index Terms-IoT Simulation; Agent Based Modelling; IoT services; Smart Objects; Internet of Things Modelling.

\section{INTRODUCTION}

The Internet of Things (IoT) [1] has become one of the most active areas in computer science and beyond in both research and business contexts. Although the existing internet is already diverse, IoT environments are more heterogeneous, connecting simple IoT devices and more advanced Smart Objects $(\mathrm{SO} s)$ with several hardware architectures, resources, software platforms, communication protocols, message formats and data repositories among others. Besides, as per forecasts, the number of internet-connected devices will reach 50 billion in the next few years [2], [3].

The engineering of software systems and applications for IoT environments reveal a number of open challenges. Particularly, the validation of such systems is complex because of three key differential characteristics of the IoT environments: the heterogeneity, volatility and the size variety. In order to validate software solutions, researchers use one or a combination of techniques that include real-world and simulations (see further background in section II). From the literature reviewed, the approaches for simulation and the models used, focus on a reduced number of the key characteristics of the IoT environments (See section IX). Other IoT test-beds, e.g.
[4], provide a real infrastructure with a defined architecture and approach for software development. As expected in shared infrastructures, there are limits to the modifications of the base software of the IoT devices, which constraints the experiments that can be carried out.

The main contribution of this paper is the definition of FABIoT, a novel agent-based model that enables the simulation of $I o T$ environments incorporating heterogeneity, volatility and medium/large quantities of IoT devices, in order to validate key properties of IoT software systems (sections III and IV). We also introduce the process for using FABIoT in the evaluation of $I o T$ software systems (section V). We evaluate FABIoT by defining a set of experiments aiming to validate workload scalability and adaptation of a concrete IoT middleware solution (section VI). Our results (section VII) show that FABIoT is an effective tool for defining and executing repeatable simulations that consider the key IoT environments characteristics and where multiple metrics can be defined and monitored (see discussion in section VIII).

\section{BACKGROUND}

\section{A. IoT Validation}

Validation in real-world testbeds is always preferred as they enable replication of the cyber-physical conditions that are present in production environments. For example the testbed used in [5] incorporates a ZigBee network with workstations, coordinators, readers and up to 200 objects with active RFID tags. The major drawback of this type of settings is the cost. Despite the low cost of hardware platforms for IoT devices, not all researchers have access to a real testbed with a sufficient number of IoT devices. Besides, the configuration, management and running of the platforms supporting these testbeds, is also time consuming. Therefore, usually, the realworld settings are constrained and scenarios lack of some or all of the key differential characteristics of IoT, namely: the heterogeneity, volatility and size variety. In the last years, a number of $I o T$ experimental research facilities with support to medium/large number of devices have appeared, offering an infrastructure for evaluating solutions atop of the offered services. These platforms offer services that reduce the effort required to evaluate a particular solution, however, the price is that application developers must conform to a particular development and operation model. e.g. IoT devices are merely data feeders and cloud infrastructure concentrates data storage and 
processing. In case of evaluating decentralized architectures that require edge processing the usefulness of these platforms is reduced. This is a problem since the $I o T$ research agenda includes the development of decentralized solutions able to run in a combination of fog, edge and cloud contexts and environments for evaluating these solutions are required.

Besides, simulations enable validation under multiple conditions, defining a model that offers a partial representation of the real-world. One of the most common uses of simulations is to validate systems' scalability to medium/large number of IoT devices, however other characteristics of heterogeneity of $I o T$ devices and volatility e.g. IoT device mobility and network topology changes, among others; are barely considered (see section IX).

\section{B. Smart Objects, Agents and Services}

One of the approaches for building the IoT vision is based on the concept of Smart Object (SO). In this approach, the SOs are the individual augmented things that together combine as a system to make IoT scenarios possible. Although the concept comes from the first decade of the century and the basis for the Smart Object-based IoT (SOb-IoT) might be from the work by Kortuem, Kawsar et al. [6] back in 2010, nowadays the SOb-IoT and the $S O$ as a concept are still under construction. The agent paradigm provides powerful abstractions with direct mapping to the $S O$ characteristics. An agent is defined by Wooldridge as "a computer system that is situated in some environment, and that is capable of autonomous action in order to meet its delegated objectives" [7]. It is clear from this definition that the agent involves a software system that works autonomously towards some objectives. In order to meet these objectives, agents sense the environment, react to it, take the initiative to carry out actions, interact and work with others.

Service and Agent computing paradigms have proven to be powerful and useful software building blocks in multiple contexts. For IoT, this is not an exception. On the one hand, Web services are particularly suitable for web application development as they provide inherent interoperability and reusability. On the other hand, agents and Multi-agent Systems (MAS) are instrumental in the development of autonomous and cooperating systems that are able to reason and proactively take actions. Inter-operability, autonomy and cooperation are fundamental to achieving the SOb-IoT vision. However, it is a challenge to ensure these paradigms are used together effectively, enhancing each other and taking advantage the existing common grounds.

\section{FABIoT MODEL OVERVIEW}

FABIoT is an Agent-based model $(A B M)$ that mimics the operation of different scale $I o T$ systems over the time. FABIoT offers the toolkit for the definition of $I O T$ environments and event-driven scenarios. The aim of FABIoT is to enable evaluation of distributed software systems that are intended to be installed in IoT devices. These systems include, for example, a collection of algorithms, services, middleware or a protocol.
Thus, FABIoT enables to create $I o T$ spaces with multiple heterogeneous, distributed and connected IoT devices. These $I o T$ devices are linked to a software library, implementing the system to evaluate. Having the IoT space and the system to evaluate, FABIoT enables to simulate different and many scenarios incorporating volatile situations that would be costly and time consuming to run in real-world settings.

In $A B M$, there are three fundamental elements: agents, environment and relationships [8]. The agents are embedded in the environment where they work autonomously during a time frame measured in ticks. In FABIoT, agents represent IoT devices, SOs, cyber physical infrastructure and humans interacting with them. SOs are IoT devices with autonomous behaviour. In our case, the environment represents a physical setting where agents are placed, it could be a residential building, a factory, a school or a neighbourhood. IoT environments defined with FABIoT incorporate several characteristics of the $I o T$ devices that are defined as parameters or variables for every experiment.

The key characteristics that make FABIoT advantageous for evaluation of IoT solutions are as follows. Note that some of these are inherited from the standard Agent-based modelling.

- Flexibility: It is designed to mimic common characteristics of the IoT environments rather than evaluating a single solution.

- Size-variety: It allows for representing small, medium and large scale environments.

- Heterogeneity-friendly: It enables definition of IoT devices with different hardware platforms, capabilities and services.

- Volatility-friendly: It enables simulation of IoT devices that are mobile, consume resources - e.g. battery, storage - or change their service offer.

- Event and Data-driven: It enables the definition of events to trigger during the simulation.

- Micro/macro levels: It provides temporal data of individual IoT devices (e.g. resources consumed) and collective system behaviour (e.g. interactions).

- Abstraction levels: It enables evaluation of software systems at different abstraction levels. e.g. software/network infrastructure and applications.

\section{FABIoT MODEL ARCHITECTURE}

In this section, we describe the key elements of the model architecture as presented in Figure 1. The main type of agent in FABIoT is the SimDevice that represents an IoT device. The Algorithm 1 shows a high level view of the main operation of a SimDevice, symbols are explained in Table I and names of functions called are self-descriptive. This agent has a hardware and software platform and is able to sense/actuate from/on the environment. The SimDevice carries out tasks which are executed using its available hardware resources. The Hardware Resource Abstraction (HRA) component enables the definition of a different mix of resources including CPU, RAM, storage, battery, sensors, actuators, embedded interfaces such as displays or keyboards, among others. The Resource Usage Model 


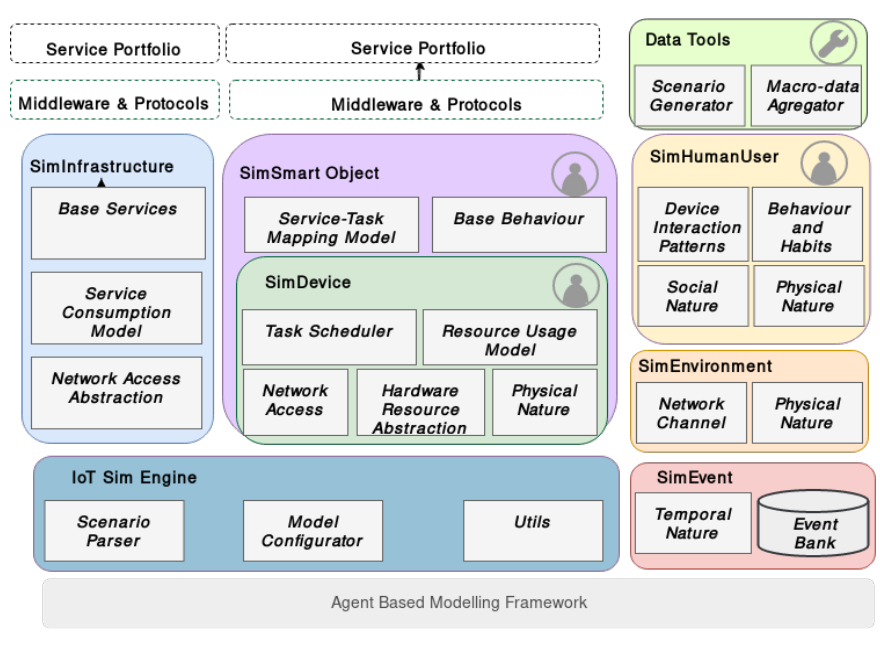

Figure. 1: FABIoT Model Architecture

(RUM) determines the way that tasks consume the resources available in the SimDevice. The Task Scheduler allocates tasks per time unit based on the hardware resources available for the device. It enables parametrization of the instructions the SimDevice runs per tick. Although the scheduling algorithm is a simplification of the real-world, it is designed to keep consistency considering the different hardware configurations. The speed of processing a task by a SimDevice, depends on the task size as well as on the device's configuration type. The more powerful the configuration type and the lower the size of the task, the quicker the SimDevice is able to process it. Battery and storage usage patterns are independent of the concrete tasks being processed. E.g. battery usage might depend on the hardware characteristics of the SimDevice such as the presence of a screen; likewise, storage might depend on the data to store, which might vary for different executions of the same task. Besides, the Physical Nature component allows for configuration of a variety of physical properties relevant to the scenario e.g. location, weight, size, etc.

On top of the SimDevice, another agent, the SimSmartObject, represents an $S O$. This is an entity that shares the hardware platform of the SimDevice but combines an autonomous behaviour and a software layer based on services. This software layer is generally the solution under evaluation. FABIoT allows for the definition of different mixes of services in a Service Portfolio. This portfolio determines which services are deployed in every $S O$ involved in a scenario. Therefore, the heterogeneity is incorporated in FABIoT at hardware and software level.

Another type of agent is the SimHumanUser, that represents the human user. This agent enables the configuration of social and physical properties of several users, as well as their behaviour within the environment and in relation to the IoT devices and SOs. These agents can trigger events or be affected by the SimDevices and SimSmartObjects. They activate functionalities under evaluation through the simulated interaction. Another component of FABIoT is the SimInfrastructure that represents any other computing resources - e.g. cloud or

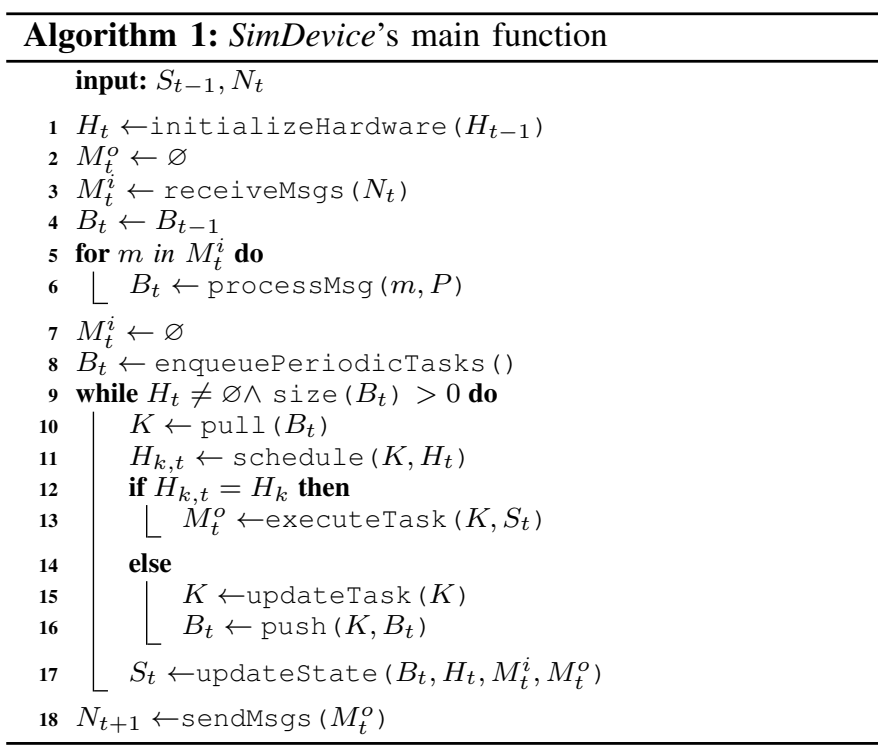

TABLE I: Symbols used in the algorithm.

\begin{tabular}{|l|r|}
\hline$B_{t}$ & SimDevice's task backlog for tick $t$. \\
\hline$H$ & $\begin{array}{c}\text { Mix of SimDevice's consumable hardware resources. } \\
\text { Defined by the 4-tuple: } H_{t}=<C_{t}, R_{t}, G_{t}, Y_{t}>, \\
\text { where } C_{t}: \text { CPU, } R_{t}: \text { RAM, } G_{t}: \text { Storage and } Y_{t} \text { : Battery. }\end{array}$ \\
\hline$H_{t}$ & Available $H$ in the tick $t$. \\
\hline$H_{k}$ & Required $H$ for the task $k$ according to resource model. \\
\hline$H_{k, t}$ & Allocated $H$ for the task $k$ in tick $t$ according to scheduling. \\
\hline$K$ & $\begin{array}{r}\text { The next task to be executed by the SimDevice. } \\
\text { Defined by } 3 \text {-tuple: } K=<k, H_{k}, F> \\
\text { where } k \text { : task id and } F: \text { Function to execute } K .\end{array}$ \\
\hline$M_{t}^{i}$ & Incoming messages to SimDevice in tick $t$. \\
\hline$M_{t}^{o}$ & Outgoing messages from SimDevice in tick $t$. \\
\hline$N_{t}$ & Simulated Network Channel's state in tick $t$. \\
\hline$P$ & Function defining the $S O$ communication protocol. \\
\hline$S_{t}$ & $\begin{array}{r}\text { SimDevice's state in the tick } t . \\
\text { Defined by the 4-tuple: } S_{t}=<B_{t}, H_{t}, M_{t}^{i}, M_{t}^{o}>.\end{array}$ \\
\hline$S_{t-1}$ & SimDevice's state in the last tick. \\
\hline
\end{tabular}

edge servers - that have powerful and sometimes unlimited resources with room to allocate and deal with more complex and resource-consuming tasks. Initially, the focus of FABIoT is in the behaviour of the IoT devices (micro level) and the whole system (macro level) rather than the infrastructure operation. Therefore, the infrastructure is abstracted in terms of services offered to the SimDevices and SimSmartObjects. The rules for usage of the available services are defined in the Service Consumption Model.

The SimEnvironment component of FABIoT defines a Network channel to which SimDevices, SimSmartObjects and SimInfrastructure have access to. The SimDevices use a standard request/response mechanism over a shared channel. The SimDevices can only send/receive messages from others connected to the same network. The message processing function, at each end, consumes hardware resources and is also considered as a task for each SimDevice.

The IoT Sim Engine is the module in charge of articulating 
all the components of a scenario and manage its execution while collecting the relevant data for analysis. This engine provides a Scenario Parser that gathers the agents, environment and events for a particular scenario from files with a specific format e.g. CSV. The Model Configurator sets up every entity of the model and triggers initialization routines.

Two separate tools ease the generation of the input data required for the model to operate. The Scenario Generator produces pseudo random data sets containing entities, agents, relationships and events. Besides, the Macro-Data Aggregator offers routines for extraction of data from the time series for each run of the scenario.

\section{Evaluation of IoT SOFTWARE Systems USING FABIoT}

This section presents the process for evaluation of an $I O T$ software system using FABIoT as depicted in Figure 2. This is a cycle inspired by the Montecarlo methods, which are useful for predicting variability of complex systems [9]. The rationale is that the behaviour of the different elements of an $I o T$ system can be modelled as a stochastic process.

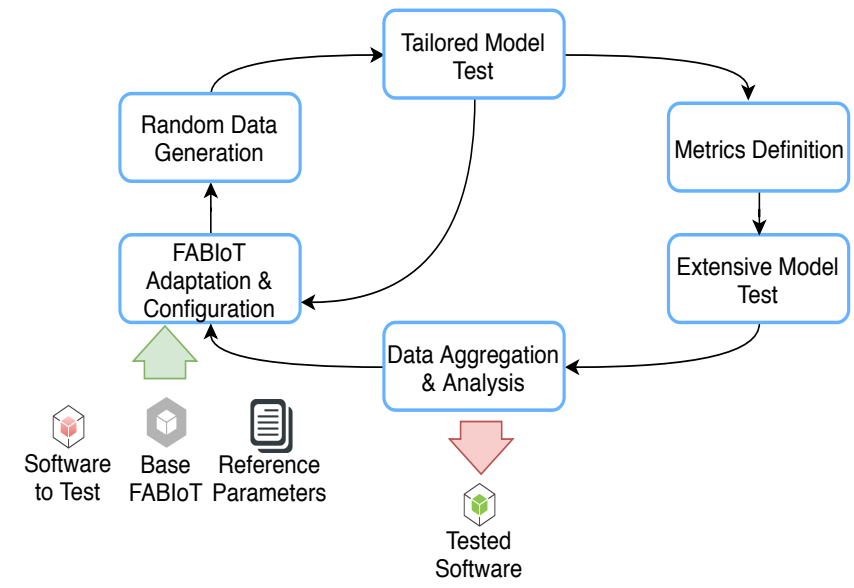

Figure. 2: $A B M$ Process for validation of $I o T$ software systems

The randomness comes from the dynamics, heterogeneity and flexibility of the IoT devices and the networks they built. Besides, there are a number of events happening during the life cycle of the IoT system, making its response variable to the specific context conditions. For example, events can be related to IoT devices (SimDevice) joining, leaving the network e.g. it runs out of battery- or sensing from the environment (SimEnvironment). We define an observation period -i.e. the time range the FABIoT model runs- during which, several events can be configured to happen. FABIoT enables to gather data of the behaviour of IoT devices, SOs and whole $I o T$ systems, working with the software system under evaluation. This is useful to evaluate its performance and identify potential issues facing unforeseen events.

The process in Figure 2 starts with the adaptation and configuration of the FABIoT base model. FABIoT works as a template offering various key elements of the $I o T$ environment but, minimally, needs to be linked to the software to test.
The result is a tailored model that is then parameterized. The parameters to configure are common to every scenario and cover the available hardware resources, the service-task mapping, the resource usage and the service consumption model. Although these parameters can be kept stable along different runs, this configuration gives the flexibility to introduce different behaviours in the SimDevices or SimSmartObjects as per requirement of the evaluation scenarios. As usual in $A B M$ models the definition of parameters requires tuning against real-world subjects. In the case of hardware resources, references are based on available real configurations and can be further adjusted with benchmark from real-world small scale tests.

The process continues with the generation of the input data for the simulation. The random-generated data covers mainly the collection of events that will be triggered during the observation period and the physical, software and hardware characteristics of the SimDevices and SimSmartObjects. The data sets for the simulation cycles are generated using the Scenario Generator tool. The data for every event varies slightly, but includes minimally the event type, the tick it should happen and the SimDevices or SimSmartObjects involved. The synthetic data enables to test the software system without the need of a wide deployment, of the solution under evaluation, among the various different real $I o T$ devices.

The next stage in the process is to test the tailored model, with a single small-scale scenario. If required, the model is re-configured and adjusted until it is ready for the extensive simulation cycles. This test provides examples of the output data, in the context of the scenarios and software under evaluation, that is used for the following step: defining the metrics relevant to study. Multiple metrics can be defined for comparing the system performance along different simulation cycles. Using FABIoT, time series data are obtained at both micro-level, i.e. the operation of particular SimDevices or SimSmartObjects, and at macro-level, the whole system behaviour. At individual level, for example, the number of resources used for a scenario provide insights about how the workload is distributed and which IoT devices are participating in a workflow. FABIoT also provides data about how SimSmartObjects manage situations when they, or their neighbours, consume the available resources. At collective level, network attributes such as latency and the overall usage of resources can also be measured. With the metrics, extensive model tests are performed, simulating the different conditions as per data generated and changing the relevant controlled parameters for the software under evaluation. Finally, the individual and collective data are aggregated and interpreted, if exit conditions for evaluation of the software are met the cycle ends, otherwise the model is adjusted and evaluated again following the same approach.

\section{IMPLEMENTATION \& EXPERIMENTS}

The aim of the experiments was primarily to determine the feasibility of FABIoT for the evaluation of an IoT software system. As per the process described in section $\mathrm{V}$, we 
need a base FABIoT model and a software to evaluate. For the former, we implemented a prototype of FABIoT using the Repast ${ }^{1}$ agent-based simulation platform. This platform provides general ABM abstractions such as agent, environment, patches, observer and a tool set for developing $\mathrm{ABM}$ models. We implemented a release of FABIoT including the SimDevices and SimSmartObjects agent types, the IoT Sim Engine, SimEnvironment, SimInfrastructure, SimEvent and the Data Tools (Section IV). For the IoT software system, we used a middleware solution that we developed in a previous work [10], [11]. This IoT middleware provides the set of abstractions for an SO-based software development and a $\mathrm{p} 2 \mathrm{p}$ communication protocol. The main functionality of the middleware enables the SOs to:

- create overlay networks where they can offer, query for and consume IoT services.

- use services for realizing activities of plans (concrete workflows) for achieving individual or cooperative goals.

- put together related services offered by each $S O$ in roles representing broader functionalities and grouping a number of services/activities (role to service proportion).

- make decisions about the SOs to cooperate with, based on the roles they play and the current context.

For demonstrating feasibility of FABIoT, we focus on the evaluation of the performance of a system of $S O s$ using the IoT mw over 50 random network configurations of 40 heterogeneous SOs (different mixes of of hardware and services). The main model parameters of the simulated environments are presented in Table II, some of these come from state-ofthe-art references - e.g. hardware configurations-, in other cases we came up with the relevant values after individual test and tuning. The observation period was 1000 ticks and each plan had 7 activities and we defined a 1 to 1 relationship between each activity and the service that realizes it. The specific conditions for each experiment are as follows:

- EX1: Using FABIoT to simulate increasing workload. It shows, from the IoT middleware perspective, how a system of SOs, using the middleware, behaves when the workload is increased and from the FABIoT perspective, how the model can be used to evaluate workload scalability of $I o T$ systems. Along the experiment, generated random events trigger from 10 to 90 plans. To see the effect of role grouping, brought by the middleware, we defined two "role to service/activity" grouping proportions: 1 to 1 (No grouping) and 1 to 5 .

- EX2: Using FABIoT model to simulate departing SOs and evaluate system adaptation/resilience. It shows, from the IoT middleware perspective, how the system of $S O s$, using the middleware, adapts when various SOs depart from the network and from the FABIoT perspective, how volatility can be simulated using FABIoT. Along the experiment, generated random events make $20 \%$ to $60 \%$, of the initial SOs, to disconnect, while keeping the

\footnotetext{
${ }^{1}$ https://repast.github.io/index.html
}

TABLE II: Main Model Parameters

\begin{tabular}{|c|c|c|c|}
\hline Name & Description & Values & Units \\
\hline \multicolumn{4}{|l|}{ Hardware } \\
\hline $\begin{array}{l}\text { Processing } \\
\text { Power }\end{array}$ & $\begin{array}{l}\text { Maximum amount (millions) of } \\
\text { instructions the } S O \text { is able to } \\
\text { process in a time unit (tick). }\end{array}$ & 1 & Mipt \\
\hline Config type & $\begin{array}{l}\text { A combination of: No. of Cores, } \\
\text { RAM and Storage, respectively. }\end{array}$ & $\begin{array}{l}\mathrm{A}(1,0.5,2) \\
\mathrm{B}(2,1,16) \\
\mathrm{C}(4,2,32) \\
\mathrm{D}(4,4,64)\end{array}$ & $\begin{array}{l}\text { Cores: Units } \\
\text { RAM: Gb } \\
\text { Storage: Gb }\end{array}$ \\
\hline$S O$ per type & Percentage of SOs per Config type & [1-140] & $\%$ \\
\hline Battery & Battery powered & {$[0,1]$} & (Discrete) \\
\hline \multicolumn{4}{|l|}{ Network } \\
\hline TTL & Time-to-live for messages & 4 & hops \\
\hline PING Freq. & How often SOs PING others. & 30 & ticks \\
\hline
\end{tabular}

workload stable. To see the effect of service density i.e. quantity of $S O S$ offering a service-, we defined two cases, one with $10 \%$ and another with $30 \%$ of the SOs offering available services.

We run the simulation cycles for each experiment in $\mathrm{ALICE}^{2}$ High Performance Computing Facility at the U. of Leicester.

\section{RESULTS}

We defined two metrics to measure the performance of the system of SOs, using the IoT middleware: Mean Query Time $(M Q T)$ and Plan Success Rate (PSR).

$M Q T$ is calculated for the plans that the whole system completes and is an indicator of how quickly the $S O s$ are able to locate other cooperating $S O s$ available. $M Q T$ is a component of the total execution time of a plan, the rest of the time depends on the power and workload of each $S O$. The $M Q T$ is calculated:

$$
M Q T=\frac{\sum_{i=1}^{n} T Q T P_{i}}{n},
$$

where $T Q T P_{i}$ is the total query time for a plan $i$ of $n$ plans completed by the system of SOs during the period of analysis. $T Q T P$ is calculated as the sum of the query time of every activity of the plan.

Plan Success Rate is calculated as follows:

$$
P S R=\frac{C P}{T P},
$$

where $C P$ is the quantity of completed plans and $T P$ is the quantity of triggered plans during one simulation cycle.

Figure 3 presents the $M Q T$ and PSR results of EX1. Each proportion of role to service/activity is identified with the different colours and shapes. The black shape shows the average in every case. The results show that the $M Q T$ decreases when the number of plans is increased. This is explained as the middleware enables the $S O$ to cache other SOs, it has cooperated with, to avoid querying for a service every time the $S O$ needs to execute an activity of a plan. It is clear that $M Q T$

\footnotetext{
${ }^{2}$ http://www2.le.ac.uk/offices/itservices/ithelp/services/hpc/alice/about
} 
values for the 1-to-5 proportion are slightly but consistently lower than the ones with no role grouping. This is explained, as the middleware enables $\mathrm{SOs}$ to reuse query results for services grouped by the same role, reducing the number of queries required and therefore the $M Q T$. The Plan Success Rate results show that the increase in the workload barely affects the system of SOs for both proportions evaluated.

Figure 4 presents the $M Q T$ and PSR results of EX2. Each service density is identified with the different colours and shapes. The black shape shows the average in every case. The $M Q T$ results show clearly two different cases when the service density is $10 \%$ and when it is $30 \%$. In the former case, the system of $S O s$ takes more time to query for services when the number of SOs departing from the network increases, showing high sensibility to these changes. The latter case is less sensible, as the $M Q T$ remains almost stable when the density is $30 \%$. The Plan Success Rate results show that the system of $S O s$ copes well with departures of $S O s$ when the density is $30 \%$, keeping the Plan Success Rate higher than $40 \%$ and mainly around $70 \%$, even with the $60 \%$ of SOs having departed. With the lower density, the Plan Success Rate is clearly lower than the previous case. These results show the middleware enables SOs to mitigate effects of volatility, by taking advantage of the available service offer.

\section{DISCUSSION}

The experiments presented, show how FABIoT was instrumental to simulate $I o T$ environments that incorporate heterogeneity, an increasing workload and volatility. FABIoT enabled us to evaluate system scalability and adaptation capabilities, determining its tolerance thresholds and identifying improvements opportunities. Although the experiments presented incorporated 40 IoT devices - a reasonable size of many IoT scenarios-, FABIoT is not size-constrained but depends on every evaluation. So far we have simulated environments with up to 140 devices. Performing these tests in real-world setting would have been not only expensive but also time consuming. Being able to generate synthetic random data enable us to test the system even in unforeseen situations. With FABIoT we did not require to deploy the system under evaluation in a real-world setting to determine how it works. Since FABIoT represents key hardware and software characteristics of the $I o T$ devices, it makes it easy to translate real-world configurations to the simulated environment. It also makes easy to generate representative situations for evaluation of the software system. We learned the importance of tuning FABIoT considering real-world reference parameters (Section V) for obtaining meaningful results. FABIoT is not intended to replace real-world test-beds but can be used as a practical complement, a previous step, in order to reduce effort, time and cost of evaluation of IoT systems.

\section{RELATED WORK}

There are several proposals for validation of IoT systems. We organize the works reviewed in two groups: real-world testbeds and simulation approaches. In this review, we explore a few examples of real-world testbeds and focus on simulation techniques, specially the existing agent-based approaches.

Real-word testbeds enable the use of a real infrastructure, usually built from a medium/large number of devices. Authors in [12] introduce FIT IoT-LAB which is a testbed with a large number and variety of nodes, distributed among 6 locations. FIT IoT-LAB enables evaluation and testing of solutions ranging from lower level protocols to advanced services and analytics in a large scale IoT environment. This testbed offers: remote access to gateways, sensors, and nodes via IP connection; monitoring of each devices power consumption and evaluation of real-time decision making in the context of 117 mobile robots. They incorporate heterogeneity at hardware level and provide tools for researchers to develop their own drivers, operating systems and applications. SmartSantander [4] is a testbed that also offers a large number of IoT devices distributed among diverse places, particularly, around the Santander city. It has a three-tier architecture including IoT nodes that sense noise and temperature, repeaters, gateways and sensors. They made possible to get the sensed data from the different nodes via different communication protocols. These platforms are robust and actively supported. As natural, these are shared by multiple researchers and they do not offer a clear approach for simulation of volatility conditions. In the real world, this is challenging as remote access and monitoring services can be compromised as well as other experiments running on the platform. In fact, FIT IoT-LAB team recognizes the importance of performing simulations before using their facilities.

Simulation approaches are wide, in most of the cases offering partial coverage of the key IoT characteristics identified in this paper. An agent-based simulation proposal using the $S O$ concept is presented in [13]. They focus on inter-SO communication while enabling definition of subnetworks and recognition of network bottlenecks. The simulation is built on top of OMNET++ platform and present different scale scenarios. Since the focus is on communication, it is not clear the approach for simulating hardware/software heterogeneity, volatility nor the reuse of the model for other experiments. Authors of [14] present a simulator enabling visualization of an architecture for the Social-IoT. Their proposal includes a GUI where connections can be defined for nodes that are part of social networks made from device, brokers and human users agents. The solution is based on a cognitive middleware offering ontologies, agents and a publish/subscribe pattern communication. Another proposal is SimpleIoTSimulator [15] which is a commercial solution where users can create IoT environments including gateways and sensors. Besides HTTP, they support some of the popular IoT protocols such as MQTT and COAP. They enable the creation of scripts with "error scenarios". One of the limitations is the lack of support to SOs that are able to work autonomously and make decisions within the IoT environment. Authors of [16], present the concept and design aspects of Sensesim. Their focus is on simulating heterogeneous and autonomous networks. Sensors are autonomous agents composed of four classes: SensorLogic, 
EX1: IoT System's Performance Evaluation With Increasing Workload Using FABloT
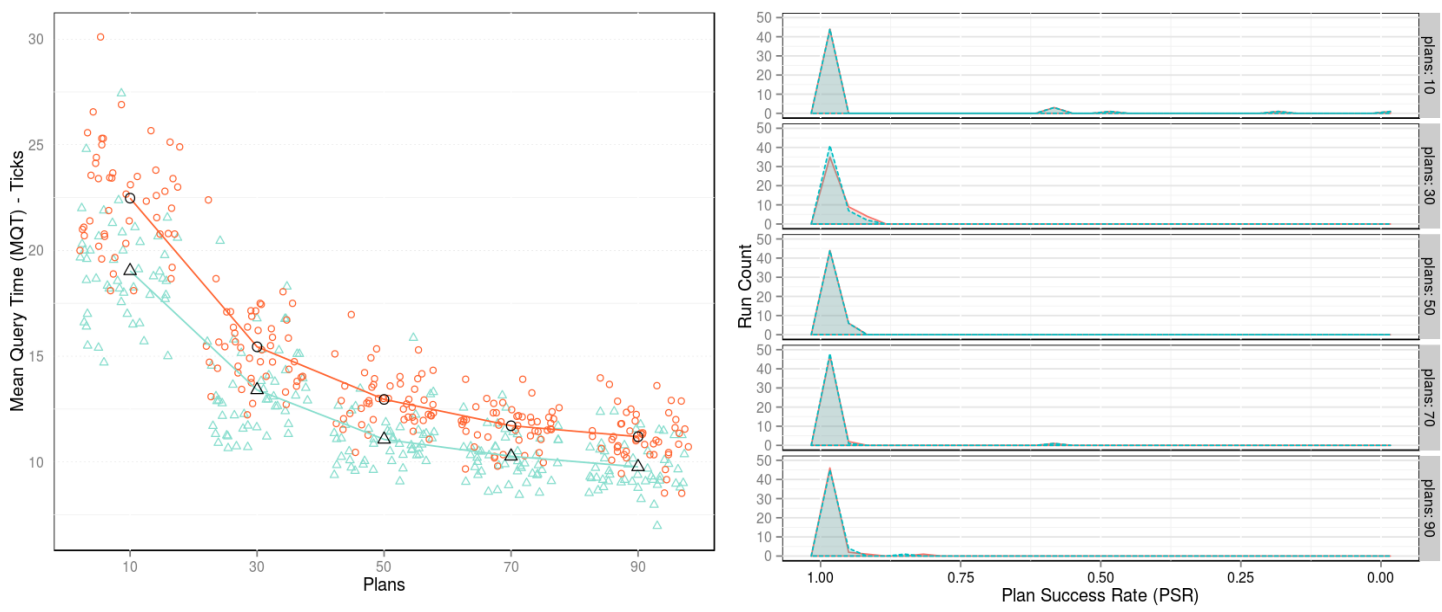

Role/Service Proportion $-\theta_{1-\text { to- } 1} \Delta_{1-\text { to-5 }}$

Figure. 3: EX1: Using FABIoT to simulate 50 different random network configurations (one per run) of an $I o T$ system with 40 heterogeneous $S O s$ facing workload increase (10 to 90 plans). It shows how: a)The $M Q T$ is inversely proportional to the workload. There is a slightly lower $M Q T$ for roles grouping five services/activities. b)The number of plans completed is not affected by the workload increase.

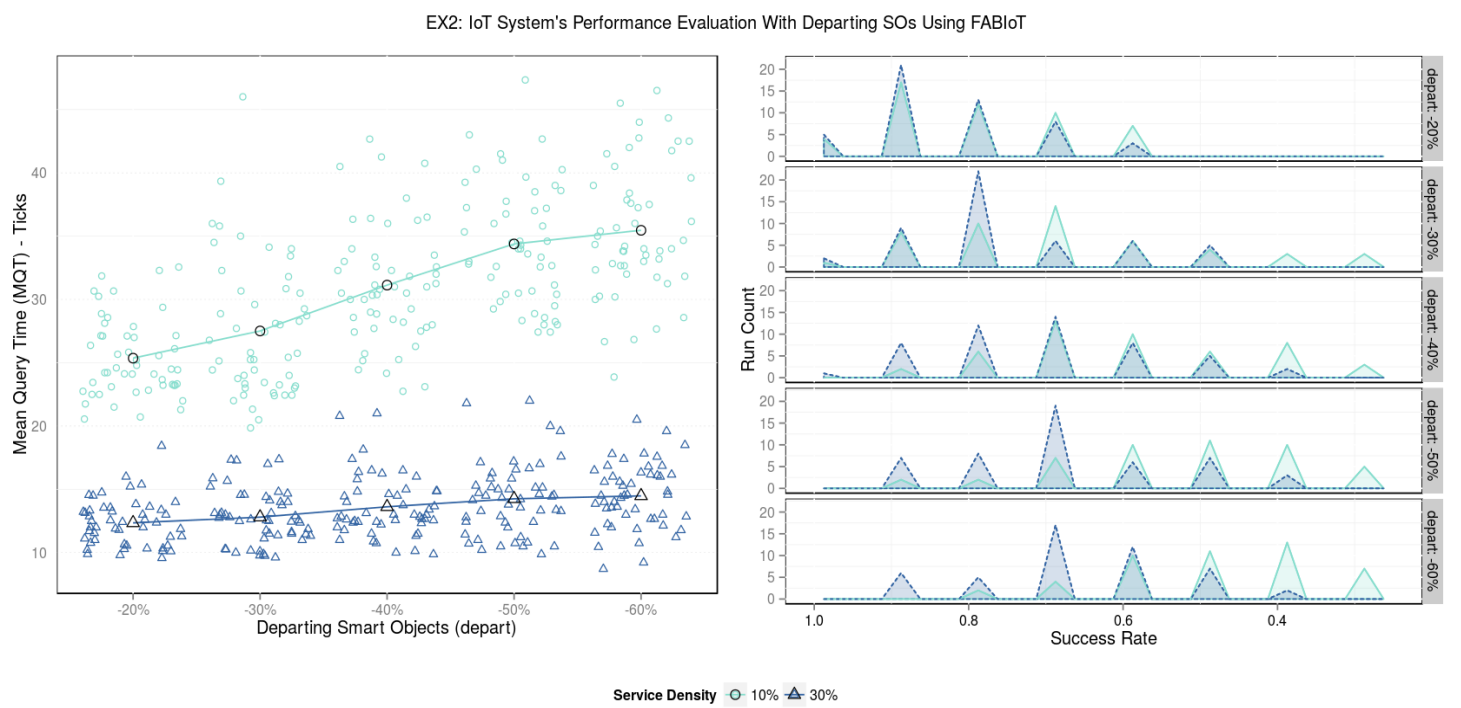

Figure. 4: EX2: Using FABIoT to simulate 50 different random network configurations (one per run) of an IoT system with 40 SOs, facing the departure of the $20 \%$ to $60 \%$ of the SOs. It shows how: a) The MQT remains stable for a high service/activity density whereas it raises for low density. b)The number of plans completed is reduced when SOs depart, particularly more sensible for low service/activity density.

SimEntity, SensorAPI and middleware. The SensorLogic class is to modify the sensor's state, the SimEntity for controlling the sensor as a simulation entity, the SensorAPI offers functions to interact with the sensor - e.g. start, stop, send messages - and the middleware that controls the execution of macro programs deployed on the sensor. A simulation platform and methodology, particularly suitable to test large IoT systems is proposed in [17]. The simulation supports distributed devices and enables simulation of IoT nodes with various network interfaces, mobility and consumption of energy.

Authors of [18] present the simulation of a smart grid system based on Multi-agent systems. They mainly focused on the device creation and energy consumption. Every device acts as an agent, enabling also the interaction with real devices and other existing agents. The paper in [19] shows an agentbased simulator built on top of the JADE Agent platform and employing Devices Profile for Web Services (DPWS). Every agent represents a SOA-ready device that uses the JADE infrastructure services to communicate to other agents. This imposes the constraint on the simulated scenarios that require a Directory Facilitator (DF) for locating others instead of using more independent $\mathrm{P} 2 \mathrm{P}$ approach. There is a layer that controls the scenarios called Superordinate logic that the user needs to perform the simulation. DPWS is also the base of 
a simulation toolkit for the design, development and testing of service-based IoT applications that is presented in [20]. This solution is based on the OASIS standard for DPWS that enables simulated devices to consume services following this standard. In this model, devices can be discovered and their operations invoked, while there is autonomous behaviour from their side. More recent proposals aim to simulate fog computing and IoT environments. One of these is iFogSim [21], which is able to simulate fog nodes and IoT environments via tuning the capabilities of devices including computational power, energy consumption, storage and communication.

Two solutions for Wireless Sensor Networks (WSN) simulations are presented in [22] and [23]. The first tool supports multiple-scale scenarios and an agent deals with events and devices, while there is an OpenStreetMap module that enables distribution of sensors, a WiSen Simulator organizes the simulation and a Solver controls the computations required for the simulation. The second proposal is an event-driven simulation with support for heterogeneity. It enables simulation of nodes with diverse energy resources, mobility models, applications and routing protocols as well as physical event simulation.

The proposals reviewed have made a good impact in research, however, we observe the lack of simulation tool addressing heterogeneity (at different levels), volatility, support to multiple scale scenarios, repeatability or induction of events, including those causing disruption to the $I o T$ system. Validation in real-world testbeds without previous simulation is not cost-efficient and can be complex to implement.

\section{Conclusion And Future Work}

This paper presents a novel agent-based model that enables the simulation of the $I O T$ system operation covering key characteristics of the IoT environments. This model allows for simulation of communication and cooperation of multiple SOs within an IoT system. We presented the architecture of our model and the simulation approach that includes the definition of randomly-triggered events that enable evaluation of IoT systems under different conditions. On top of this model, multiple metrics can be monitored and defined according to the experiments to perform. We evaluated our model with a series of experiments for the validation of an IoT middleware that is embedded in simulated Smart Objects.

On the basis of the promising findings presented in this paper, future work will involve simulation of IoT environments highlighting differences between Fog and Cloud computing contexts. Besides, we will assess the use of real data sets, when available. We would like to use data gathering patterns about device resource usage, e.g. energy consumption.

\section{REFERENCES}

[1] L. Atzori, A. Iera, and G. Morabito, "The internet of things: A survey," Computer networks, vol. 54, no. 15, pp. 2787-2805, 2010.

[2] H. Sundmaeker, P. Guillemin, P. Friess, and S. Woelfflé, "Vision and challenges for realising the internet of things," Cluster of European Research Projects on the Internet of Things, European Commision, vol. 3, no. 3, pp. 34-36, 2010.
[3] C. Perera, A. Zaslavsky, P. Christen, and D. Georgakopoulos, "Context aware computing for the internet of things: A survey," IEEE communications surveys \& tutorials, vol. 16, no. 1, pp. 414-454, 2014.

[4] L. Sanchez, L. Muñoz, J. A. Galache, P. Sotres, J. R. Santana, V. Gutierrez, R. Ramdhany, A. Gluhak, S. Krco, E. Theodoridis et al., "Smartsantander: Iot experimentation over a smart city testbed," Computer Networks, vol. 61, pp. 217-238, 2014.

[5] D. Zhang, L. T. Yang, M. Chen, S. Zhao, M. Guo, and Y. Zhang, "Realtime locating systems using active rfid for internet of things," IEEE Systems Journal, vol. 10, no. 3, pp. 1226-1235, 2016.

[6] G. Kortuem, F. Kawsar, V. Sundramoorthy, and D. Fitton, "Smart objects as building blocks for the internet of things," IEEE Internet Computing, vol. 14 , no. 1, pp. 44-51, 2010.

[7] M. Wooldridge, Introduction to Multiagent Systems, 2nd ed. Glasgow: Wiley \& Sons, Ltd, 2009, vol. 30.

[8] C. M. MacAl and M. J. North, "Tutorial on agent-based modelling and simulation," Journal of Simulation, vol. 4, no. 3, pp. 151-162, 2010.

[9] B. Dodson, P. C. Hammett, and R. Klerx, Probabilistic Design for Optimization and Robustness for Engineers. Chichester, England; Hoboken, NJ: John Wiley \& Sons, Ltd, 2014.

[10] M. E. P. Hernandez and S. Reiff-Marganiec, "Autonomous and Self Controlling Smart Objects for the Future Internet," 2015 3rd International Conference on Future Internet of Things and Cloud, pp. 301-308, 2015.

[11] M. E. Perez Hernandez and S. Reiff-marganiec, "Towards a software framework for the autonomous internet of things," in 2016 IEEE 4th International Conference on Future Internet of Things and Cloud (FiCloud), 2016.

[12] C. Adjih, E. Baccelli, E. Fleury, G. Harter, N. Mitton, T. Noel, R. Pissard-Gibollet, F. Saint-Marcel, G. Schreiner, J. Vandaele, and T. Watteyne, "Fit iot-lab: A large scale open experimental iot testbed," in 2015 IEEE 2nd (WF-IoT), Dec 2015, pp. 459-464.

[13] G. Fortino, W. Russo, and C. Savaglio, "Agent-oriented modeling and simulation of iot networks," in Computer Science and Information Systems (FedCSIS), 2016 Federated Conference. IEEE, 2016, pp. 14491452.

[14] P. Kasnesis, L. Toumanidis, D. Kogias, C. Z. Patrikakis, and I. S. Venieris, "Assist: An agent-based siot simulator," in 2016 IEEE 3rd (WF-IoT), Dec 2016, pp. 353-358.

[15] simpleiotsimulator: the internet of things simulator. Accessed: 201802-19. [Online]. Available: http://www.smplsft.com/

[16] M. Dyk, A. Najgebauer, and D. Pierzchala, "Sensesim: An agent-based and discrete event simulator for wireless sensor networks and the internet of things," in 2015 IEEE 2nd World Forum on Internet of Things (WFIoT), Dec 2015, pp. 345-350.

[17] G. Brambilla, M. Picone, S. Cirani, M. Amoretti, and F. Zanichelli, "A simulation platform for large-scale internet of things scenarios in urban environments," in Proceedings of the First International Conference on IoT in Urban Space. ICST, 2014, pp. 50-55.

[18] S. Karnouskos and T. N. De Holanda, "Simulation of a smart grid city with software agents," in Computer Modeling and Simulation, 2009. EMS'09. Third UKSim European Symposium on. IEEE, 2009, pp. 424-429.

[19] S. Karnouskos and M. M. J. Tariq, "Using multi-agent systems to simulate dynamic infrastructures populated with large numbers of web service enabled devices," in Autonomous Decentralized Systems, 2009. ISADS'09. International Symposium on. IEEE, 2009, pp. 1-7.

[20] S. N. Han, G. M. Lee, N. Crespi, N. Van Luong, K. Heo, M. Brut, and P. Gatellier, "Dpwsim: A simulation toolkit for iot applications using devices profile for web services," in Internet of Things (WF-IoT), 2014 IEEE World Forum on. IEEE, 2014, pp. 544-547.

[21] H. Gupta, A. Vahid Dastjerdi, S. K. Ghosh, and R. Buyya, "ifogsim: A toolkit for modeling and simulation of resource management techniques in the internet of things, edge and fog computing environments," Software: Practice and Experience, vol. 47, no. 9, pp. 1275-1296, 2017.

[22] K. Mehdi, M. Lounis, A. Bounceur, and T. Kechadi, "Cupcarbon: A multi-agent and discrete event wireless sensor network design and simulation tool," in 7th International ICST Conference on Simulation Tools and Techniques, Lisbon, Portugal, 17-19 March 2014. ICST, 2014, pp. 126-131.

[23] G. Chelius, A. Fraboulet, and E. Fleury, "Wsnet: a modular event-driven wireless network simulator," 2006. 